\title{
Micromachined atomic force microprobe with integrated capacitive read-out
}

\author{
J Brugger, R A Buser and N F de Rooij \\ Institute of Microtechnology, University of Neuchâtel, Breguet 2, 2000 Neuchâtel, \\ Switzerland
}

\begin{abstract}
We developed a micromachining process for the fabrication of highly sensitive capacitor probes to be used for displacement measurement of an atomic force cantilever. The capacitive structure consists of two adjacent single-crystal silicon beams, one carrying a sharp tip for the force interaction, the other being the counter-electrode. The air gap of $1.5 \mu \mathrm{m}$ separating the two electrodes is obtained by removal of the oxide in between by selective etching. The capacitance has a typical value of $\simeq 0.2 \mathrm{pF}$. Forces acting on the tip induce a bending of the cantilever and change the capacitance which can be detected by electronic circuits.
\end{abstract}

\section{Introduction}

Using capacitive variation to sense physical displacements represents an interesting alternative to optical, tunneling or piezoresistive methods. Sensors that use single-crystal silicon as highly elastic material and capacitance change as the readout principle are known to be reliable and accurate [1]. Additionally, thermal silicon dioxide provides good electrical insulation and allows, in combination with highly doped silicon, the formation of capacitors with low losses. Micromachined silicon acceleration sensors have been presented with a plate area of about $4 \times 4 \mathrm{~mm}^{2}$ and a gap of $7 \mu \mathrm{m}$, thus forming a capacitance of $C=20 \mathrm{pF}$. When associated with a CMOS detection circuit this sensor yields a theoretical resolution of $40 \mathrm{aF}$ at $1 \mathrm{~Hz}$ [2].

In the field of atomic force microscopy (AFM) where a force-induced lever bending has to be measured accurately, detection methods using optical, tunneling and capacitive techniques are most common. For the capacitance technique wires were used as force-sensing levers and aluminium foils as capacitor plates $\left(300 \times 300 \mu \mathrm{m}^{2}\right)$ with a gap of $1 \mu \mathrm{m}$, yielding a static capacitance of about $C \simeq 1 \mathrm{pF}$. With the achieved sensitivity of $\simeq 10^{-6} \mathrm{pF}$, a variation of the inter-plate distance can be resolved down to $0.01 \AA$ [3]. Another paper reported a similar sensitivity utilizing a micromachined silicon strip with a side length of $2.3 \mathrm{~mm}$ attached via field-assistance bonding to a glass plate and separated from two counter electrodes. This configuration has been applied in a bidirectional AFM [4]. All these constructions are assembled by individual pieces which is a major disadvantage. With a view to an integrated microprobe, a piezoresistive cantilever [5] and an electrostatically driven poly-silicon scan-tip obtained by sacrificial layer techniques with interferometrical readout [6] have been published.

We investigated the feasibility of highly sensitive capacitive cantilever sensors using micromachining techniques, with the advantage of the integration of the sensing and readout element, which forms an interesting class of reproducible low-cost sensors. Another advantage of this technique is the possibility to produce microprobe arrays. In this paper we describe the fabrication steps of an integrated capacitance AFM sensor.

\section{Capacitive structure}

The two-electrode capacitance structure consists of two adjacent single-crystal silicon beams, one carrying a sharp tip being the measurement cantilever, the other being the counter electrode. Both are connected to a carrier piece of wafer thickness, but separated by an intermediate silicon dioxide $\left(\mathrm{SiO}_{2}\right)$ layer of about $1.5 \mu \mathrm{m}$ thickness (figure 1). The capacitance is given by $C=\varepsilon A / d$ and is the sum of the sensing capacitance $C_{s}$ (between the electrodes) and the parallel capacitance $C_{\mathrm{p}}$ (of the carrier dioxide), thus $C_{\text {tot }}=C_{\mathrm{s}}+C_{\mathrm{p}}$. When $A_{\mathrm{s}}=700 \times 30 \mu \mathrm{m}^{2}$ and $d=1.5 \mu \mathrm{m}$ are the area and gap of the sensing probes and $\varepsilon_{0}=8.85 \times 10^{-12} A_{s} / V_{\mathrm{m}}$, the capacitance $C_{\mathrm{s}}$ is of the order of $0.3 \mathrm{pF}$. In order to decrease the undesirable $C_{\mathrm{p}}$, the anchoring surface $A_{\mathrm{p}}$ of the cantilever to the holder piece can be minimized to some tens of $\mu \mathrm{m}$ width, thus forming a $C_{\mathrm{p}}$ of about $10 \mathrm{pF}$, with $\varepsilon=\varepsilon_{0} \varepsilon_{\mathrm{r}}\left(\varepsilon_{\mathrm{r}}=3.9\right.$ for thermal $\mathrm{SiO}_{2}$ ).

The mechanical behaviour of the silicon cantilever is determined by its geometrical dimensions of width $w$, height $h$ and length $l$ respectively. The spring constant is given by $k=E w h^{3} / 4 l^{3}\left(E=1.69 \times 10^{11} \mathrm{~N} \mathrm{~m}^{-2}\right)$ and can 


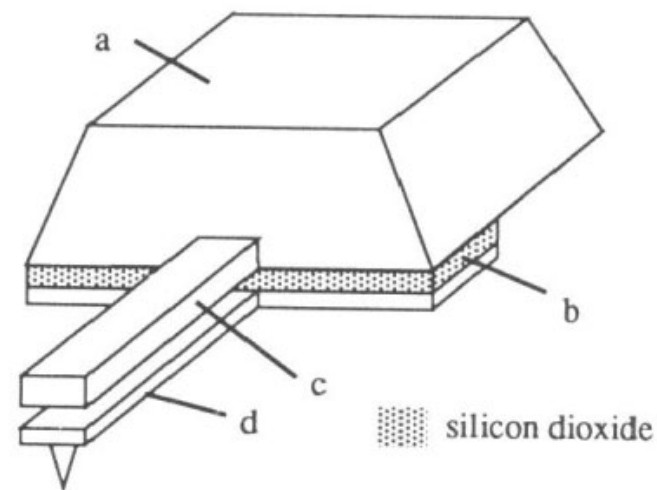

Figure 1. AFM microprobe with integrated capacitive read-out detection: (a) carrier, (b) insulating $\mathrm{SiO}_{2},(c)$ counter electrode, $(d)$ cantilever with tip.

be varied in our case from 0.1 to $100 \mathrm{Nm}^{-1}$ by using different layouts. The counter electrode is considerably thicker $(>20 \mu \mathrm{m})$ than the sensing cantilever $(\simeq 5 \mu \mathrm{m})$ in order to make the non-sensing electrode become less sensitive to changes in the electrical field during the measurement. For the design of a practical system a

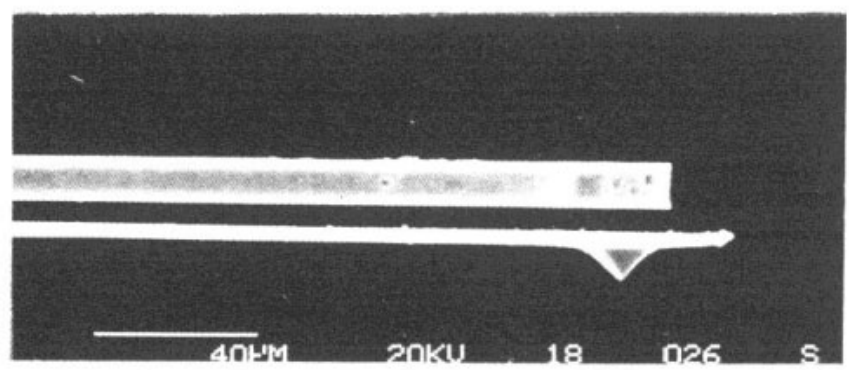

Figure 2. SEM micrograph of a micromachined capacitive microprobe: upper part, counter-electrode; lower part, cantilever with tip.

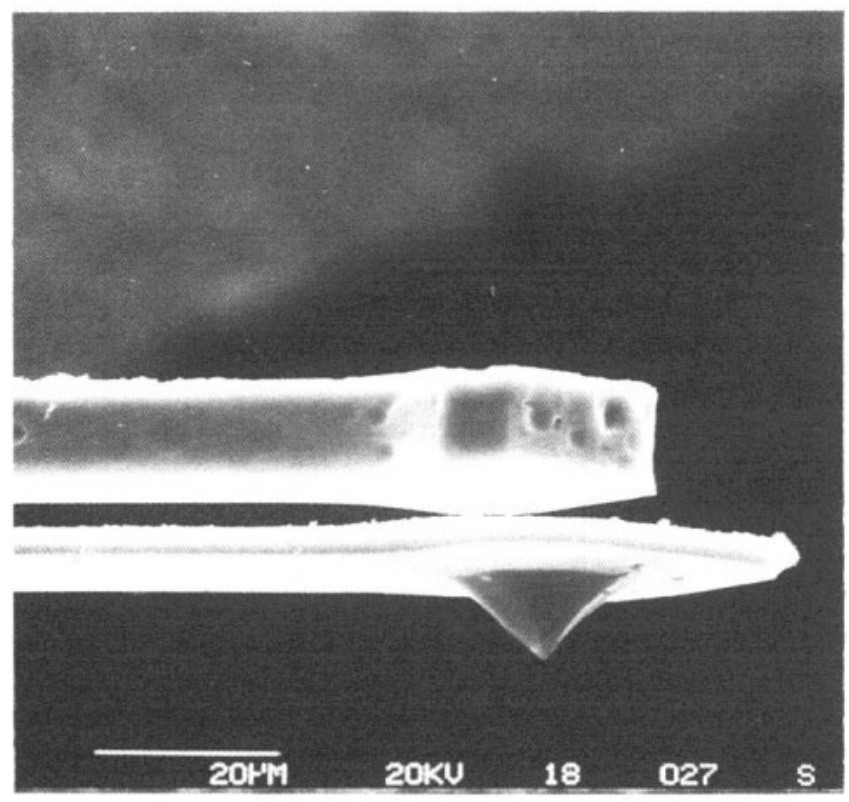

Figure 3. Close-up of the microfabricated AFM capacitive sensor. compromise must be made between the opposing demands of a high sensitivity readout $(\rightarrow$ thinner cantilever and smaller gap) and the prevention of snapping $(\rightarrow$ thicker cantilever and larger gap) [4].

Figures 2 and 3 show the result obtained by the fabrication steps described hereafter.

\section{Microfabrication}

The process of the integrated capacitive AfM microprobe is based upon the microfabrication steps of simple passive cantilevers as described elsewhere [7], extended by additional steps such as silicon fusion bonding (SFB) and additional reactive ion etching (RIE), as illustrated in figure 4 , where the dotted line in figure $4(b)$ illustrates the site of the cantilever and tip in the starting bulk material.

a)

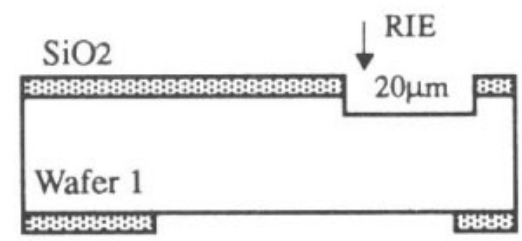

b)

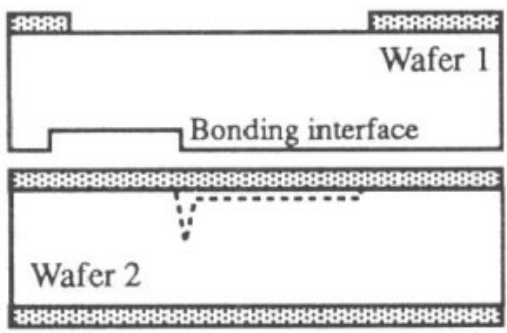

c)

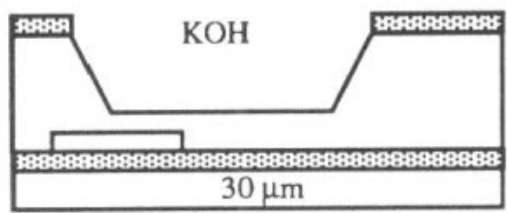

d)

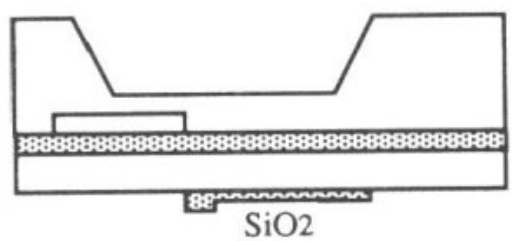

e)

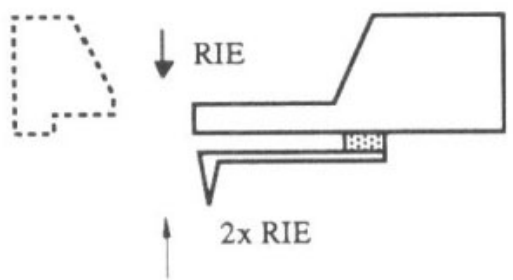

Figure 4. Microfabrication steps for the integrated capacitive readout AFM microprobe. 
First, wafer \#1 (i.e. the carrier wafer with the counter electrode) was RIE etched to form $20 \mu \mathrm{m}$ deep trenches [8], the future counter electrodes were protected by the $\mathrm{SiO}_{2}$ and photoresist (figure 4(a)). Wafer \#2 (with the cantilever and tip) was SF-bonded to the prestructured wafer \#1-now up-side down-thus forming selected bond areas. Both wafers were cleaned and the surfaces hydrolyzed before bringing into contact. SFB in an oxidation furnace at about $1100^{\circ} \mathrm{C}$ for $4 \mathrm{~h}$ was performed sealing the two surfaces together. The undesirable $\mathrm{SiO}_{2}$ which was formed in the $\mathrm{KOH}$-etch windows of wafer \#1 during this step was removed by re-masking and by buffered HF (BHF) etching this side. Simultaneously the $\mathrm{SiO}_{2}$ on the backside of wafer \#2 was etched for subsequent processing (figure $4(b)$ ). A membrane of about $25 \mu \mathrm{m}$ thickness was formed on both wafers by timecontrolled $\mathrm{KOH}$ etching (figure $4(c)$ ). Two additional mask steps for the cantilever and tip were performed on the re-oxidized surface of wafer \#2 (figure $4(d)$ ). Then three RIE steps were performed successively, first from the backside of wafer \# 1 to pierce through the membrane and to form the counter electrode, second and third to pre-shape the cantilever and the tip. Wet etching in $\mathrm{HNO}_{3}: \mathrm{HF}: \mathrm{CH}_{3} \mathrm{COOH}$ released the cantilever from the membrane and formed a high protruding silicon tip. Finally by extended BHF etching, the silicon dioxide between the two capacitance electrodes was removed, forming a uniform gap between the two plates (figure $4(e)$ ). The individual sensor is attached by thin silicon beams to the wafer-chip and can easily be removed.

\section{Conclusion}

We have presented a silicon micromachining process for the fabrication of an atomic force microprobe designed for a capacitive readout, thus combining the advantageous mechanical properties of single-crystal silicon cantilevers and integrating it with a highly sensitive readout mechanism. Dry and wet etching as well as silicon fusion bonding have been successfully combined to fabricate two adjacent single-crystal silicon cantilevers forming the capacitance structure. A next step would be the integration of some electronic components for the readout in combination with a low-temperature bonding technique, e.g. sputtered glass.

\section{Acknowledgments}

We would like to acknowledge S Jeanneret and P-A Clerc for technical assistance. This work has been supported by the Swiss National Science Foundation.

\section{References}

[1] Herens W C 1986 Application of capacitance techniques in sensor design J. Phys. E: Sci. Instrum. $19897-906$

[2] Rudolf F, Jornod A, Bergqvist J and Leuthold H 1990 Precision accelerometers with $\mu \mathrm{g}$ resolution Sensors Actuators A 21-23 297-302

[3] Göddenhenrich T, Lemke H, Hartmann $U$ and Heiden C 1990 Force microscope with capacitive displacement detection $J$. Vac. Sci. Technol. A $8383-7$

[4] Neubauer G, Cohen S R, McClelland G M, Horne D and Mate C M 1990 Force microscopy with a bidirectional capacitance sensor Rev. Sci. Instrum. 61 2296-308

[5] Tortonese M, Yamada H, Barett R C and Quate C F 1991 Atomic force microscopy using a piezoresistive cantilever Digest of technical papers, Transducers '91 (San Francisco, 1991) pp 448-51

[6] Koñg L C, Oni B G and Wise K D 1990 A micromachined silicon scan tip for an atomic force microscope Digest of technical papers, Hilton Head 90

[7] Brugger J, Buser R A and de Rooij N F 1992 Silicon cantilevers and tips for scanning force microscopy Sensors Actuators A 34 193-200

[8] Linder C, Tschan T and de Rooij N F 1992 Deep dry etching of silicon-a novel micromachining tool Sensors Mater. 3 311-24 